\title{
Clinical Results And Review of Techniques of Lumbar Endoscopic Unilateral Laminotomy With Bilateral Decompression (LE-ULBD) for Lumbar Stenosis
}

\author{
Hyeun Sung $\mathrm{Kim}^{1}$, Pang Hung $\mathrm{Wu}^{1,2}$, Il-Tae Jang ${ }^{1}$ \\ ${ }^{1}$ Department of Neurosurgery, Nanoori Gangnam Hospital, Seoul, Spine Surgery, Seoul, Republic of Korea \\ ${ }^{2}$ Department of Orthopaedic Surgery, National University Health System, Jurong Health Campus, Singapore
}

Corresponding Author:

Hyeun Sung Kim, MD, PhD

Department of Neurosurgery, Nanoori

Hospital Gangnam, Seoul 731, Eonju-ro,

Gangnam-gu, Seoul, Republic of Korea

Tel: $+82-2-6003-9767$

Fax: +82-2-3445-9755

Email: neurospinekim@gmail.com

Received: January 27, 2021

Revised: March 19, 2021

Accepted: March 22, 2021

\begin{abstract}
Uniportal Lumbar Endoscopic decompression can be performed through transforaminal and interlaminar route. Interlaminar lumbar endoscopic unilateral laminotomy for bilateral decompression allows good decompression of central and lateral recess of the stenotic lumbar spine region. Both over the top decompression approach and under the ligamentum flavum decompression approach method has been recently described with differing principles and approaches despite achieving the same target in decompression of spinal canal through uniportal interlaminar endoscopic route. The authors aim to share their experience and thoughts on the 2 described approaches. Retrospective clinical cohort evaluation of patients who underwent LEULBD were performed from January 2018 to December 2019 The cohort of 278 cases of LEULBD with mean age of 64 years old were evaluated. Complica- tion rate is $3.6 \%$ and reoperation was 3.6\%, mean VAS improvement at 1 weeks, 3 months and final follow up were $3.06 \pm 0.66$, $2.50 \pm 0.86$ and $2.17 \pm 0.91$ respectively, $p<0.001$ and ODI impro- vement at 1 weeks, 3 months and final follow up were $31.87 \pm 5.02,27.91 \pm 6.31$ and $25.32 \pm 6.44$ respectively. Lumbar Endoscopic Unilateral Laminotomy Bilateral Decompression could achieve good clinical outcomes and low rate of complications with thorough understanding of endoscopic anatomy.
\end{abstract}

Key Words: Spinal stenosis, Endoscopic spine surgery, Lumbar endoscopic unilateral laminotomy for bilateral decompression, Ligamentum flavum, Foraminal ligament, Lumbar endoscopic unilateral laminotomy for bilateral decompression

\section{INTRODUCTION}

The incidence of spinal stenosis is increasing due to aging population. The demand for minimally invasive surgery, less perioperative morbidities and early mobilization drives the development of endoscopic spine surger ${ }^{6,12)}$. However, until endoscopic burrs were developed, endoscopic decompression was practically not easy. Endoscopic decompression developed into two routes: transforaminal and interlaminar approaches (Figure 1).

The evolution of endoscopic spine surgery (ESS) leads to an increasing trend of more Lumbar Endoscopic Unilateral Laminotomy For Bilateral Decompression (LEULBD) being performed for spinal stenosis and related conditions. There are several technical approaches described for LEULBD to achieve the same target of spinal decompression ${ }^{2,4,9,10)}$. The key differences in the described techniques can be divided into docking and identification of key bony landmark, approach to lamina, method of removal of ipsilateral and contralateral ligamentum flavum. While there are differences in details, there are benefits and risks inherent to each of the various described techniques. In this technical review, we describe the key differences in the over the top decompression and under the ligamentum flavum decompression approach of LEULBD, which recently coined as Outside-in by Kim et al. ${ }^{4)}$ and Inside-out by Lim et al. ${ }^{8)} \mathrm{A}$ retrospective analysis of clinical outcome of our cohort of LEULBD patient was performed.

\section{Analysis of Our Series of Lumbar Endoscopic Unila- teral Laminotomy with Bilateral Decompression}

This retrospective study was reviewed by institutional review board of Nanoori Hospital, Seoul, Republic of Korea. Retrospective clinical evaluation of patients who met indications of lumbar endoscopic unilateral laminotomy with bilateral decompression (LEULBD) were included in the study. These are patients who were included had either (1) foraminal stenosis, (2) herniated 

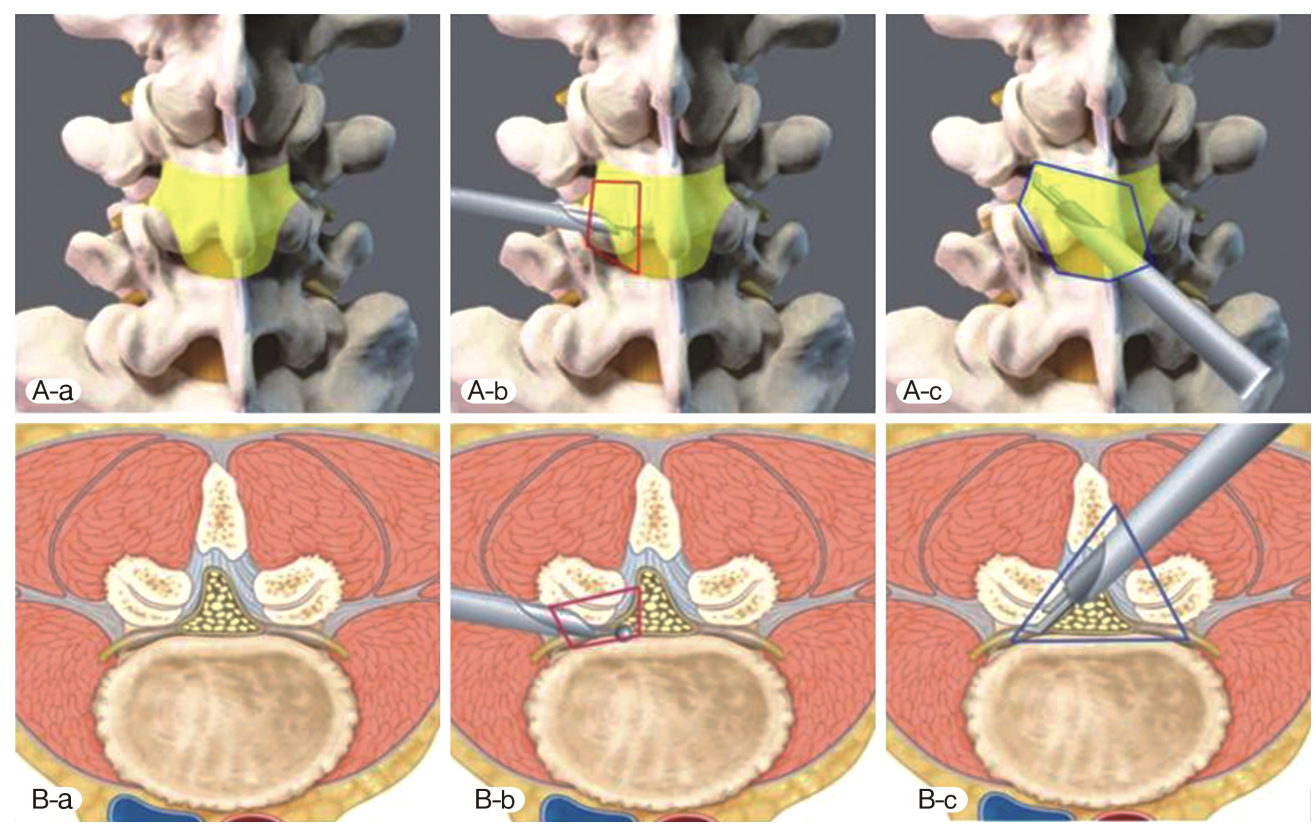

Figure 1. Endoscopic decompression coverage area. Yellow shaded area represented the attachment of ligamentum flavum. A-b, B-b: Transforaminal decompression, A-c, B-c: Interlaminar decompression. Blue line represented the area of decompression in Lumbar Endoscopic Unilateral Laminotomy For Bilateral Decompression.

lumbar disc with concurrent ligamentum hypertrophy, (3) spinal stenosis and (4) ossified ligamentum flavum who failed minimal six weeks of conservative treatment with minimum preoperative Visualized Analog Scale of 5 and Oswestry Disability Index of 50 were included in the study. We excluded revision surgery, patients who had concurrent tumor, infection instability of lumbar spine and fractures. Collection of pre and postoperative clinical data of Clinical Visual Analog Scale and Oswestry Disability Index was done retrospectively in clinical consultation at 1 week post-operative, 3 months post-operative and final follow up.

\section{Statistical Analysis}

Clinical data was analyzed with SPSS version 18 statistical analysis software (IBM corporation, New York). The continuous variables were expressed as mean and standard deviation (SD). The paired t-test was used clinical visual analogue scale (VAS), Oswestry Disability Index (ODI) measured at pre-operative, 1 weeks postoperative, 3 months post-operative and final follow up repor ted by the patients were analysed. A value of $(p<0.05)$ considered significant within the cohort.

\section{RESULTS}

278 patients (159 female and 119 male) underwent single or multiple levels LEULBD were included in the study. Mean age of the cohort of patients were 64 (10-89). 18 patients had foraminal stenosis, 5 had herniated lumbar disc with concurrent ligamentum hypertrophy, 1 had ossified ligamentum flavum and 254
Table 1. Clinical visual analog scale and oswestry disability index improvement of our cohort of patients who underwent lumbar endoscopic unilateral laminotomy with bilateral decompression

\begin{tabular}{l|c|c|c}
\hline \hline & Mean & Std. Deviation & $p$-value \\
\hline VAS improvement at 1 weeks & 3.05 & 0.66 & $p<0.001$ \\
VAS improvement at 3 months & 2.50 & 0.86 & $p<0.001$ \\
VAS improvement at final follow up & 2.17 & 0.91 & $p<0.001$ \\
ODI improvement at 1 weeks & 31.87 & 5.02 & $p<0.001$ \\
ODI improvement at 3 months & 27.91 & 6.31 & $p<0.001$ \\
ODI improvement at final follow up & 25.32 & 6.44 & $p<0.001$ \\
\hline
\end{tabular}

had spinal stenosis. 154 patients underwent 1 level decompression, 105 patients had 2 levels, 17 had 3 levels and 3 had 4 levels LEULBD decompression. 10 cases of complications were observed making up 3.6\% of cases, 8 had incidental durotomy, 1 had foot drop postoperatively and 1 had weakness of power 4 in L5 distribution after operation. The patient with foot drop had dural tear with prolapsed and incarcerated nerve root which required open revision of dural defect. The residual 8 dural tear were trea ted with patch blocking repair technique without sequelae ${ }^{5)}$.

Preoperative, postoperative 1 week, 3 months and final follow up mean VAS were 7.5(5-10), 3.1(1-6), 2.5(1-5) and 2.2(1-6) respectively.

Preoperative, postoperative 1 week, 3 months and final follow up mean ODI were 73(52-88), 32(16-56) , 28(16-58) and 25(6-56) respectively (Table 1).

75 patients had excellent, 194 good, 8 fair and 1 poor MacNab's score results. 


\section{DISCUSSION}

Incidence of various degenerative conditions of lumbar spine such as spinal stenosis and herniated nucleus pulposus leading to the need for decompression is increasing ${ }^{12)}$. The gold standard treatment is laminectomy along with flavectomy to increase spinal canal volume. The approach can be done open, tubular microscopic and endoscopic decompression. Key to success is good orientation of surgical anatomy and adequate bony and flavum removal with preservation of facet joint to conserve stability of spine ${ }^{1)}$. The concept of LEULBD is not new. Recently, Lim et al and Kim et al coined the terms, inside out (under the ligamentum flavum decompression approach) ${ }^{8)}$ and outside-in (over the top decompression) ${ }^{4)}$ approach to describe the endoscopic handling of ligamentum flavum. With the under the ligamentum flavum decompression approach focus on usage of endoscopic Kerisson Rongeurs removing the ligamentum flavum with the lamina bone together, entering the epidural space early in the procedure. While Over the top decompression approach focus on endoscopic drilling of lamina leaving the ligamentum flavum intact till the last stages of the procedures. Both authors highlighted the pros and cons of their technique. It is contentious

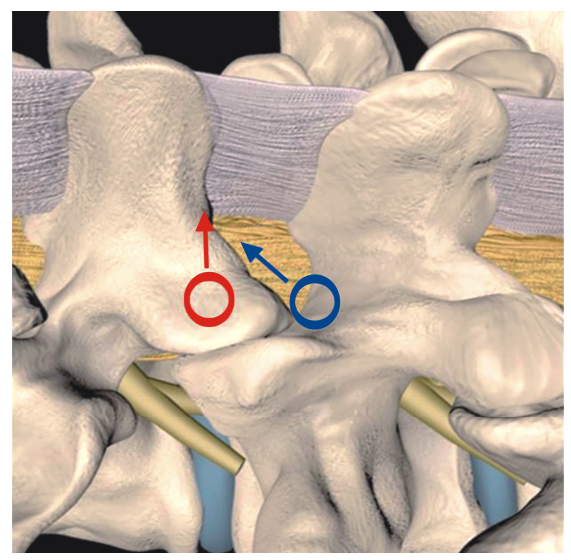

Figure 2. Docking Point: Red: translaminar approach, Blue: sublaminar approach. that LEULBD is divided into under the ligamentum flavum decompression approach( inside-out) or over the top decompression approach (outside-in) as most surgeons do a hybrid of both technique. This review aims to elaborate the subtle differences of these 2 approaches with similar ultimate goal of adequate decompression and conservation of facet joint.

\section{Technical Review of Lumbar Endoscopic Unilateral Laminotomy with Bilateral Decompression}

\section{1) Docking and Identification of Key Bony Landmarks \\ (1) Docking}

Under the ligamentum flavum decompression approach Technique docked at the spinolaminar junction of the cephalad lamina'. While Over the top decompression approach technique docked at the laminofacet junction between caudal lamina and cephalad inferior articular process, which the authors termed as "V" point ${ }^{4}$. This variation in docking is important factor consideration in removal of cephalad attachment of flavum from cephalad lamina as the spinolaminar docking tends to remove the cephalad flavum attachment via a translaminar approach while "V" point docking remove the cephalad flavum attachment via sublaminar approach $^{2,4,9)}$ (Figures 2-4).

\section{Ipsilateral Cephalad Lamina Resection to Free Up Ligamentum Flavum Attachment}

IO technique used a combination of endoscopic Kerrison punch and drills from the inferior border of the cranial lamina to the detachment of cephalad ligamentum flavum ${ }^{9}$. In over the top decompression approach, our authors tend to use endoscopic drill to drill the ventral portion of inferior border of the cranial lamina till the flavum attachment is loose (Figure 5A and $B)^{4)}$. The angle of resection of the cephalad lamina is in a more vertical angle with less caudal cranial inclination as compared to over the top decompression In technique. There is a higher likelihood of full thickness bony removal of cephalad lamina to expose the flavum in under ligamentum flavum approach technique. The nature of caudal cranial inclination in over the top

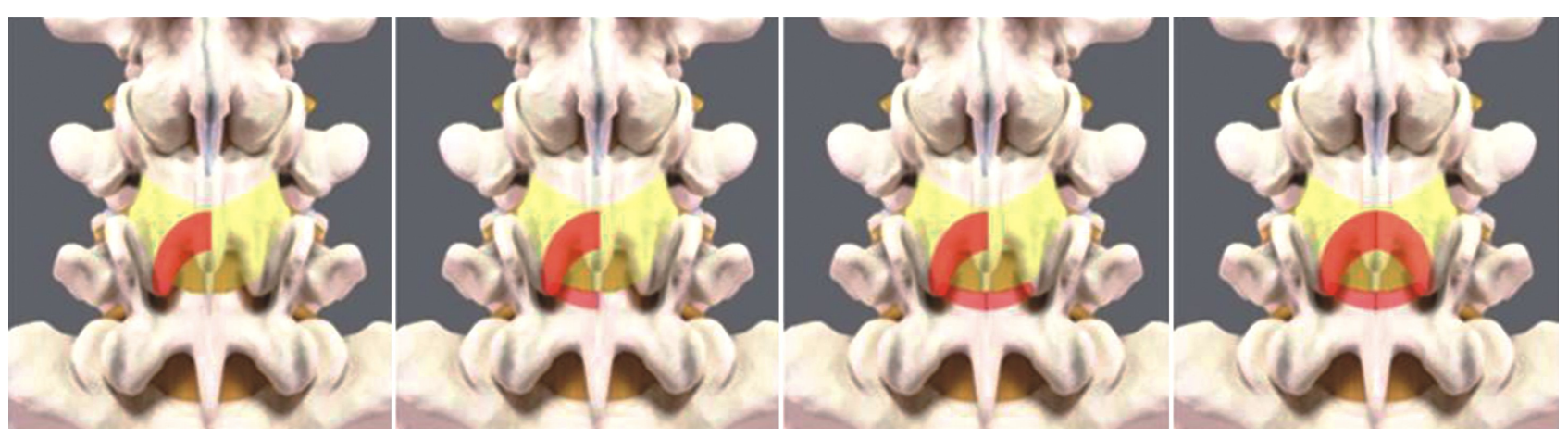

Figure 3. Endoscopic drilling sequence of lumbar full endoscopic, unilateral approach, bilateral decompression over the top decompression approach. 
decompression approach to cephalad lamina will tend to have a wedge shape lamina resection with the dorsal portion of the lamina preserved when cephalad ligamentum flavum is detached. There is no literature to suggest that translaminar and sublaminar approach to cephalad ligamentum flavum attachment makes any clinical difference in outcomes ${ }^{2,4,9,10}$.

\section{Ipsilateral Caudal Lamina Resection to Free Up Ligamentum Flavum Attachment}

The use of saline irrigation and creation of epidural space and early identification of neural structures is key in under ligamentum flavum approach to caudal lamina. Full thickness flavum is removed by Kerrison rongeur by biting against caudal lamina bone. Usage of endoscopic curette to lift the caudal ligamentum flavum attachment from caudal lamina is another alternative. Epidural bleeding is encountered early in the procedure during neural elements exposure. Hemostasis is performed with radiofrequency ablation. In over the top decompression approach caudal lamina is drilled or curetted to detach superficial ligamentum flavum from the deep layer of ligamentum flavum (Figure 5C). The caudal lamina is then drilled under endoscopic vision to thin out the caudal lamina till the edge of deep caudal ligamentum flavum attachment.

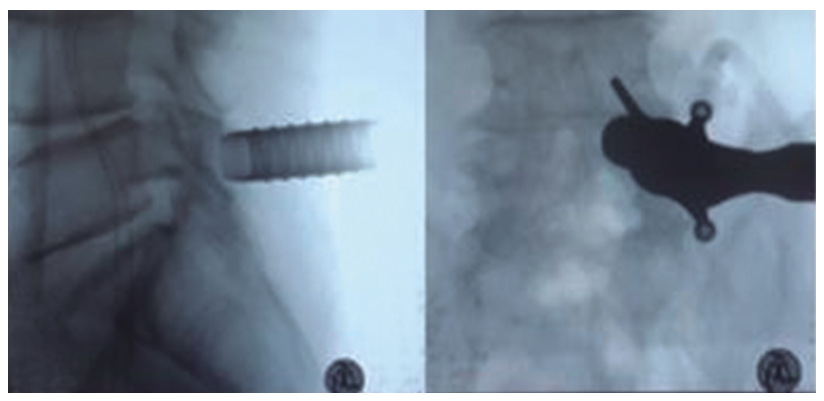

Figure 4. Sublaminar Approach Docking. For right LEULBD of $L 4 / 5$, right $L 4 / 5$ laminofacet junction were docked after serial dilation.

\section{1) Ipsilateral Facet}

In the under ligamentum flavum approach, early medial laminotomy and early piecemeal removal of detached ligamentum flavum show the traversing nerve root early which is followed laterally to remove the lateral margin of ligamentum flavum attachment to the superior articular facet (SAP). Medial aspect of inferior articular facet (IAP) is resected with endoscopic drill or Kerrison to expose the SAP required to be resected. In Ol approach, early exposure of SAP is done by directly docking on the laminofacet junction and drilling the medial portion of IAP just enough to expose the medial portion of SAP. SAP is then drilled from medial to lateral and caudal to cephalad direction till the lateral margin of ligamentum flavum is detached. While early exposure of lateral margin of traversing nerve root in under ligamentum flavum approach gives guidance of the facet resection required to detached flavum, the use of endoscopic drill to resect SAP which is deployed in over the top decompression cases are not advisable. In over the top decompression approach, the preservation of deep ligamentum flavum to be removed at the end of procedure is key in acting as a shield to prevent inadvertent durotomy.

\section{2) Contralateral Cephalad and Caudal Lamina}

Base of spinous processes and contralateral laminae were undercut using endoscopic drill and endoscopic Kerrison rongeur until the contralateral cephalad ligamentum flavum is detached. Process is similar in both under ligamentum flavum and over the top decompression approaches except that in under ligamentum flavum approachthe dura is exposed early while in over the top decompression approach the ipsilateral flavum is still remained attached until all bony decompression is completed.

\section{3) Contralateral Facet}

In under ligamentum flavum approach, water irrigation, blunt dissectors were used to separate a plane between the contralateral ligamentum flavum and the epidural fat and dura and probe on the contralateral SAP and separate adhesion from dura.
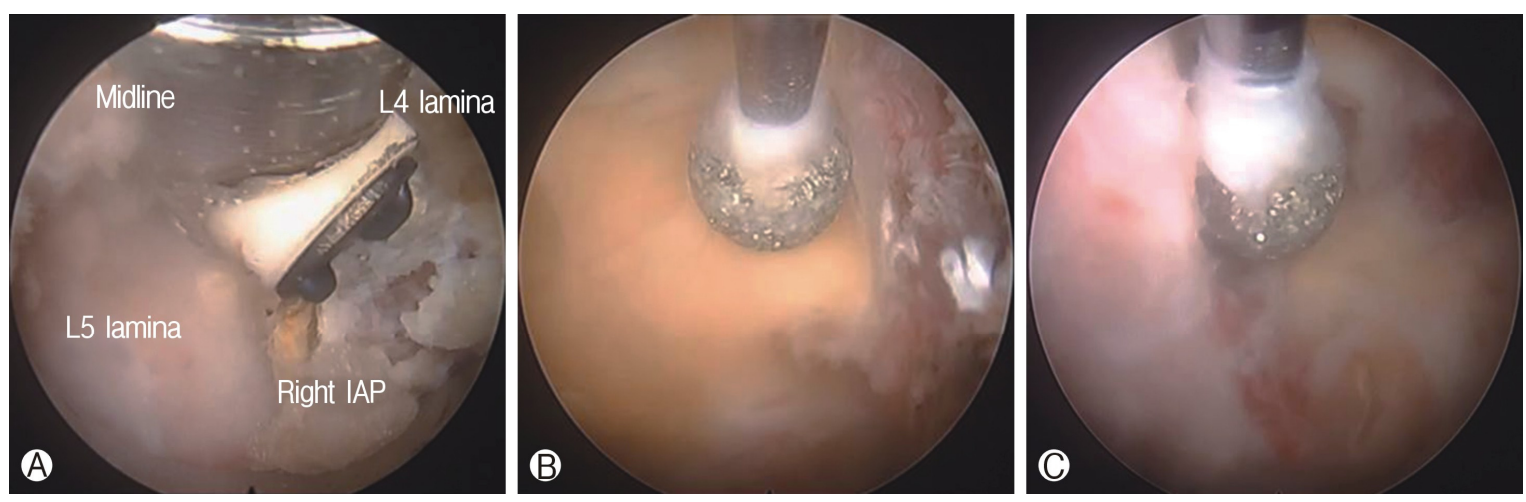

Figure 5. (A): Exposure of $V$ point of laminofacet junction of right $L 4 / 5 L E U L B D$. (B): Ipsilateral lamniotomy with deep layer of ligamentum flavum protecting the neural elements from endoscopic drill. (C): Caudal laminotomy with endoscopic drill till ligamentum flavum caudal edge. 

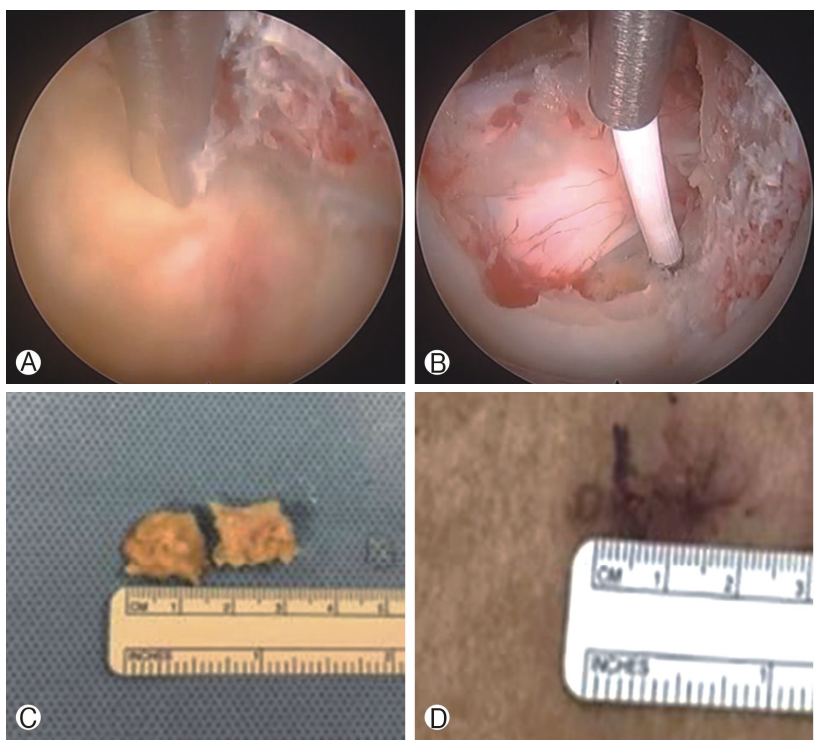

Figure 6. (A): Using a probe, ligamentum flavum attachment of the contralateral L4 lamina is taken down bluntly. (B): Completion of decompression showing decompressed neural elements. (C) 2 pieces of ligamentum flavum removed en bloc with outside in ligamentum flavum resection technique. (D): wound size of right LEULBD L4/5.
Careful piecemeal removal of contralateral flavum using Endoscopic Kerrison punches the contralateral ligamentum flavum from SAP attachment from underneath the flavum pointing Kerisson in dorsal direction away from neural elements. In Ol approach, the contralateral deep layer of flavum is preserved till late. Medial portion of SAP is resected under endoscopic vision with endoscopic drill and Kerrison rongeur till the lateral ligamentum flavum attachment is loose (Figure 6A). After ligamentum flavum was removed, hemostasis with low energy radiofrequency probe on epidural vessels (Figure 6B). Typically 2 blocks of thickened ligamentum flavum could be removed en bloc (Figure 6C) through a small $1 \mathrm{~cm}$ wound (Figure 6D).

Figure 7 Clinical photograph and pre and postoperative MRI images of Over the top decompression approach Lumbar Endoscopic Unilateral Laminotomy For Bilateral Decompression

\section{4) Dangers and Potential Complications}

In both under ligamentum flavum and over the top decompression approaches, most common complications are dura tear, neck pain/headache due to water irrigation pressure and incomplete decompression. Dura tear can happen in 3-10\% of cases. Small incidental durotomy can be repaired by patch blocking repair technique using gelfoam and tachosi ${ }^{5}$. Neckache
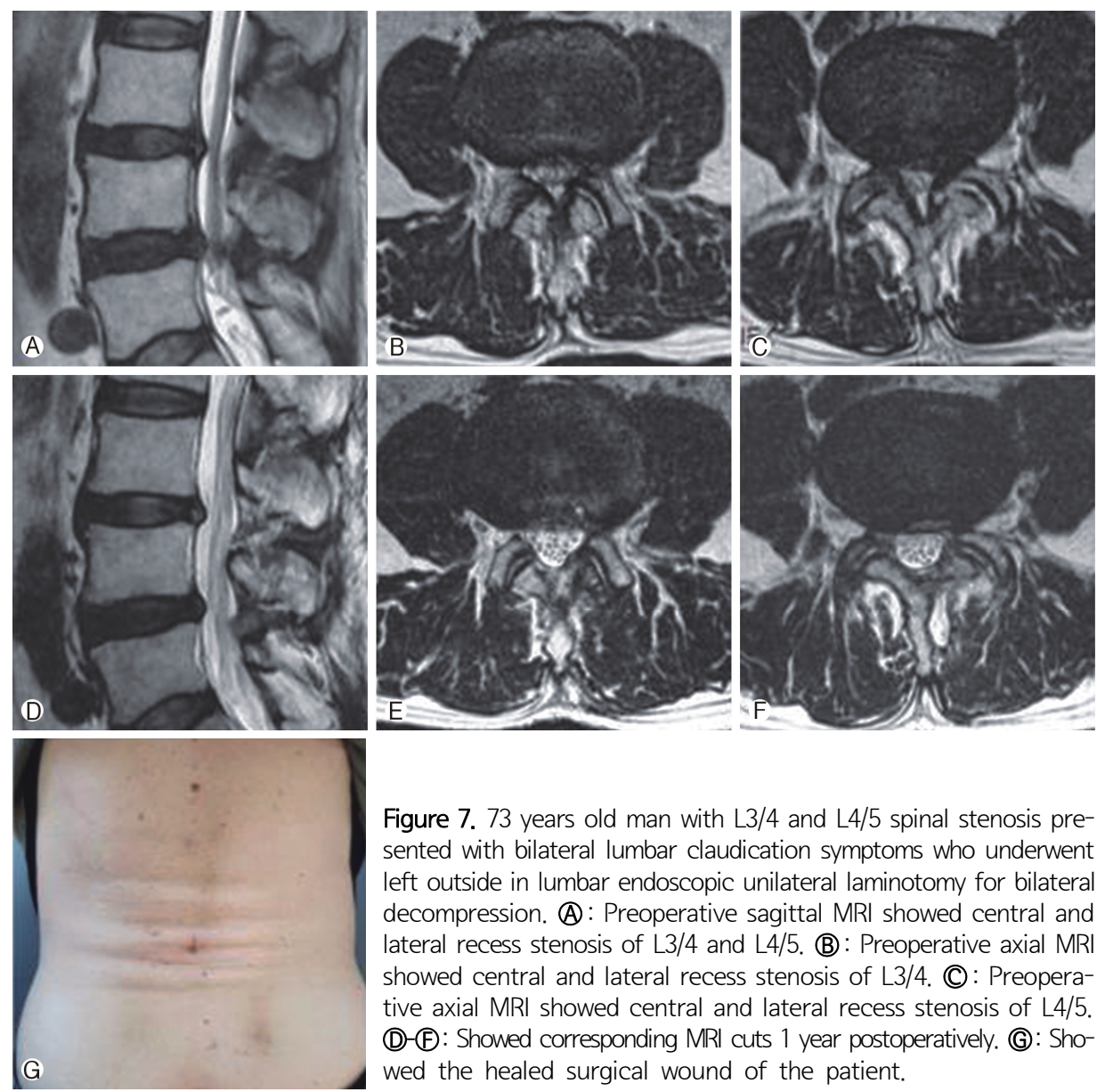

Figure 7. 73 years old man with $L 3 / 4$ and $L 4 / 5$ spinal stenosis presented with bilateral lumbar claudication symptoms who underwent left outside in lumbar endoscopic unilateral laminotomy for bilateral decompression. (A): Preoperative sagittal MRI showed central and lateral recess stenosis of $L 3 / 4$ and $L 4 / 5$. (B): Preoperative axial MRI showed central and lateral recess stenosis of L3/4. (C): Preoperative axial MRI showed central and lateral recess stenosis of $L 4 / 5$. (D)- $($ ): Showed corresponding MRI cuts 1 year postoperatively. (G): Showed the healed surgical wound of the patient. 
and headache can be prevented by limiting the duration of surgery with high irrigation pressure. Optimal irrigation pressure is in the range of $25-45 \mathrm{mmHg}$ with the mean of $30 \mathrm{mmHg}^{3,4}$. A short duration of high irrigation pressure at no more than $70-80 \mathrm{mmHg}$ can be tolerated. Incomplete decompression can happen in any decompression surgery. The decision on how much decompression is necessary requires careful preoperative planning. Fluoroscopic guidance and navigation can help to overcome the steep learning curve in determining the amount of decompression required yet not causing spinal instability ${ }^{11)}$. Ligamentum flavum en bloc resection and the exposure of lateral margin of the neural elements can help to endoscopically examine the completeness of decompression. Spinal instability is a potential complication in any spinal decompression surgery. Studies show that endoscopic surgery may have a tendency to preserve more facet joints and potentially lower risk of instability ${ }^{1,7)}$. More long term data is required to establish this plausible theory. Small wounds in LEULBD may decrease infection risk compared to open surgery, it is advisable for an experienced team of scrubbed nurses, surgeons and radiographers to enhance safety and decrease infection rate in LEULBD.

In our cohort of patients who underwent the "over the top decompression approach" version of LEULBD. They showed statistically significant improvement at various time point of follow up, with a relatively low rate of complications of 3.5\%.

The learning curve of LEULBD is steep, the results of good clinical outcomes and low complication rate is achieved after the authors overcame the learning curve with regular practice of spinal endoscopy and ability to handle complications using endoscopic technique. In our cohort of patients, we achieved statistically significant improvement at 1 weeks, 3 months and final follow up for both Visual Analog Scale and Oswestry Disability Index.

\section{CONCLUSION}

A thorough understanding of ligamentum flavum attachment and the technical approaches in lumbar endoscopic unilateral laminotomy for bilateral decompression can achieve good clinical outcomes.

\section{ACKNOWLEDGMENTS}

Dr. Hyeun-Sung Kim and Dr. Pang Hung Wu contributed equally to this work as first authors.

\section{CONFLICT OF INTEREST}

Dr. HS Kim and Dr. PH Wu are editorial board members of the journal but were not involved in the peer reviewer selection, evaluation, or decision process of this article. There are no other potential conflicts of interest relevant to this article to declare.

\section{REFERENCES}

1. Heo DH, Lee DC, Park CK: Comparative analysis of three types of minimally invasive decompressive surgery for lumbar central stenosis: Biportal endoscopy, uniportal endoscopy, and microsurgery. Neurosurg Focus 46:E9, 2019

2. Ito F, Ito Z, Shibayama M, Nakamura S, Yamada M, Yoshimatu H, Takeuchi M, Shimizu K, Miura Y: Step-by-Step Sublaminar Approach With a Newly-Designed Spinal Endoscope for UnilateralApproach Bilateral Decompression in Spinal Stenosis. Neurospine 16:41-51, 2019

3. Kang MS, Park HJ, Hwang JH, Kim JE, Choi DJ, Chung HJ: Safety Evaluation of Biportal Endoscopic Lumbar Discectomy: Assessment of Cervical Epidural Pressure During Surgery. Spine (Phila Pa 1976) 45:E1349-e1356,

4. Kim H-S, Wu PH, Jang I-T: Lumbar Endoscopic Unilateral Laminotomy for Bilateral Decompression Outside-In Approach: A Proctorship Guideline With 12 Steps of Effectiveness and Safety. Neurospine 17:S99-S109, 2020

5. Kim HS, Raorane HD, Hung WP, Heo DH, Sharma SB, Jang IT: Incidental Durotomy during Endoscopic Stenotic Lumbar Decompression (ESLD): Incidence, classification and proposed management strategies. World Neurosurg 140:612-617, 2020

6. Kim HS, Wu PH, Jang I-T: Current and Future of Endoscopic Spine Surgery: What are the Common Procedures we Have Now and What Lies Ahead? World Neurosurgery 140:642-653, 2020

7. Lee C-W, Yoon K-J, Ha S-S: Comparative Analysis between Three Different Lumbar Decompression Techniques (Microscopic, Tubular, and Endoscopic) in Lumbar Canal and Lateral Recess Stenosis: Preliminary Report. BioMed Research International 2019:60784696078469, 2019

8. Lim KT, Meceda EJA, Park C-K: Inside-Out Approach of Lumbar Endoscopic Unilateral Laminotomy for Bilateral Decompression: A Detailed Technical Description, Rationale and Outcomes. Neurospine 17:S88-S98, 2020

9. Lim KT, Nam HGW, Kim SB, Kim HS, Park JS, Park CK: Therapeutic Feasibility of Full Endoscopic Decompression in Oneto Three-Level Lumbar Canal Stenosis via a Single Skin Port Using a New Endoscopic System, Percutaneous Stenoscopic Lumbar Decompression. Asian Spine J 13:272-282, 2019

10. Ruetten S, Komp M, Merk H, Godolias G: Surgical treatment for lumbar lateral recess stenosis with the full-endoscopic interlaminar approach versus conventional microsurgical technique: A prospective, randomized, controlled study. J Neurosurg Spine 10:476485, 2009

11. Wu PH: Early Career Challenges in Setting Up an Endoscopic Spine Surgery Practice. World Neurosurgery 144:264-269, 2020

12. Wu PH, Kim HS, Jang I-T: A Narrative Review of Development of Full-Endoscopic Lumbar Spine Surgery. Neurospine 17:S20S33, 2020 